\title{
Inhalt
}

- Qualitative Analyse der Kohlenstoffverbindungen. . . . . . 1

Nachweis von Kohlenstoff und Wasserstoff . . . . . . . . 1

Verkoblung 1. Oxydation mit Kupferoxyd, $\mathrm{CuO} 1$.

Nachweis von Stickstoff . . . . . . . . . . . . . . . 2

Methode von Lassaignz 2. Methode von Will und Varkentrapr 2. Methode ron KJELDaHL 3.

Nachweis der Halogene . . . . . . . . . . . . . . . 3

BeILstern sche Probe 3. Glahen mit Kalk 3.

Nachweis von Schwefel.

Methode von CariUs 4. Erwărmen mit Natron 4. Glühen mit Natrium 5.

II. Die allotropen Formen des Kohlenstoffis . . . . . . . . 5

Graphit 5. Holzkohle 5. Knochenkohle 5. Entfärbung von Lösungen durch Knochenkoble $b$. Absorption von Bleisalzen durch Knochenkoble 6. Entfernung von riechenden Stoffen mittels Knochenkoble 6.

III. Arbeitsmethoden

Fraktionierte Destillation 6. Dampfdestillation 7. Ausziehen mit I.ösungsmitteln 8. Schmelzpunktbestimmung 8. Bestimmung des Siedepunkts 8 . Kristallisation 9.

IV. Gesättigte Kohlenwasserstoffe

Darstellung von Methan, $\mathrm{CH}_{4}$ 9. Eigenschaften des Methans 9. Destillation von Petroleum 10. Entzlundlichkeit der Petroleumdämpfe I0. Geringe Reaktionsfahigkeit der gesättigten Kohlenwasserstoffe 10.

V. Alkohole, $\mathrm{C}_{\mathrm{n}} \mathrm{H}_{2 \mathrm{n}+1} \cdot \mathrm{OH}$

Elnwirkung von Natrium auf Alkohol 11. Fuselöl 11. Wein 11. Entwicklung von Wărme bel der Mischung von Alkohol und Wasser 11. Nachweis von Wasser in Alkohol 12. Trennung von Wasser und Alkohol 12. Oxydation des Âthylalkohols 12 .

VI. Alkylhaloide, Ester, Äther und Mercaptan

Äthylcblorid 13. Äthylschwefelsăure 13. Diăthylăther, $\left(\mathrm{C}_{2} \mathrm{H}_{3}\right)_{2} \mathrm{O}$ 14. I.̈̄slichkelt von Ather und Wasser Ineinander 14. Eigenschaften des Äthers 15. Äthylmerkaptan 15.

VII. Amine und Nitroverbindungen . . . . . . . . . . . 15

Amine . . . . . . . . . . . . . . . . . . . . . 15

Methylamin 15. Karbylaminreaktion 15. Senfolreaktion 16. Einwirkung auf Kupfersalze 16. Einwirkung von salpetriger Săure 16. Nitrosamine 17. Dimethylamin und Trimethylamin 17.

Nitroverbindungen . . . . . . . . . . . . . . . . .

Natriumnitromethan 17. Nitrolsăurereaktion 18. Reduktion der Nitroverbindungen 18 . 
VIII. Nitrile und Isonitrile

Darstellung 18. Verseifung der Nitrile 18.

IX. Gesättigte Säuren, $\mathrm{C}_{\mathrm{n}} \mathrm{H}_{2 n} \mathrm{O}_{2}$, und Ester

Ameisensăure 19. Zersetzung von Merkuri- und von Silberformiat 19. Einwirkung von konzentrierter Scbwefelsäure auf Ameisensăure 20. Oxydation von Ameisensăure durch Kalfumpermanganat 20. Kakodylreaktion der Essigsăure 20. Bestăndigkeit der Essigsäure gegen Oxydationsmittel 21. Silberacetat 21. Nachwefs von Essigsãure 21. Oxydation von Äthylalkohol zu Essigsăure 22. Darstellung von Methan aus Acetat 22. Verseifung von Butter 22. Calcium- und Blelsalze der höheren Fettsäuren 23. Flachtige Fettsăuren 23. Höhere Fettsăuren 23. Seife 23. Emulgierende Wirkung von Selfe 24. Keinigende Wirkung von Seife 24. Essigsăureăthylester 24. Verseifungsgeschwindigkeit des Essiggỡureăthylesters 24. Löslichkeit des Essigesters in Wasser 25.

X. Aldehyde und Ketone. . . . . . . . . . . . . . . 25 Aldehyde . . . . . . . . . . . . . . . . . . . . 25

Acetaldehyd 25. Silberspiegelprobe 25. Aldehydbarz 26. ScBifrache Reaktion 26. Bildung von Formaldehyd 26. Einwirkung von Formalin anf Eieralbumin 26. Einwirkung ron Formaldebyd auf Gelatine 26. Formalin und Natronlauge 26.

Ketone .

Aceton 27. Löslichkeit von Quecksilberoxyd in Aceton 27. Additionaprodukt von Aceton und Natriumbisulfit 27. Abscheidung von Aceton aus einer wäBrigen lösung 27. Oxydation von Aceton 28.

XI. Ungesättigte Kohlenwasserstoffe .

Olefine.

Äthylen 28. Leuchtkraft der Äthylenfamme 28. Eivwirkung von Brom auf .̈thylen 28. Nachwels einer Doppelbindung nach BAEYER 29. Fxplosion einer Mischung von Äthylen und Bsuerstoff 29. Ungesättigte Kohlenwasserstoffe in Leuchtgas 29. Doppelbindung im Amylen. 29.

Acetylen

Darstellung von Acetylen 29. Acetylenkupfer und Acetylensilber 30.

XII. Einbasische ungesättigte Säuren

Doppelbindung im Mandelöl 30. Ölsăure aus Mandelöl 31. Überfubrung in Elaidinsăure 31. Schmelzen von Ölsăure mit Natron 31. Bleioleat 31 .

XIII. Chloroform und Jodoform

Darstellung von Chloroform 32. Bildung von Chloroform aus Trichloressigвăure 32. Einwirkung von Kali auf Chloroform 32. Überfohrung von Chloroform in Kaliumeyanid 33. Chloroform und Silbernitrat 33. Jodoformreaktion zum Nachweis von Alkohol 33.

XIV. Glyzerin

Darstellung von Allylalkohol 33. Nachweis von Glyzerin 34. Einwirkung von Alkalien auf Kupfersalze bei Gegenwart von Glyzerin 34. ScвотTrNBAUMANX sche leaktion zum Nachweis von Hydroxylgruppen 34.

XV. Gesättigte zweibasische Säuren .

Darstellung von Oxalsăure aus Holz 34. Ubberfuhrung von Formiaten in Oxalate 35. Calcium-, Blei- und Kupferoxalat 35. Zersetzung von Calclumoxalat durch Hitze 35. Zersetzung von Blei- und Kupferoxalat durch Hitze 36 . Einwirkung von konzentrierter Schwefelsăure anf Oxalsăure 36. Komplexe Oxalate 36. Wirkung des Lichtes auf EDEk sche Lösung 37. Oxalsäuredimethylcster 37. Oxalsăurediāthylester 37 . Oxamid 38.

Bernsteinsäure

Baryumsuccinat 38 . Basisches Ferrisuccinat 38 . Succininild 38. 
XVI. Oxysäuren oder Alkoholsăuren . . . . . . . . . . . 39

Milchsäure . . . . . . . . . . . . . . . . . . . . . . . 39

Zersetzung von MLlclusăure durch verdonnte Scbwefelsăure 39. Zersetzung von Milchsăure durch konzentrlerte Schwefelsăure 39. Oxydierung von Milchsăure 39.

Weinsäure

Kaliumtartrat 39. Eisenammoniumtartrat 40. FEuLiNe sche Lösung 40. Verhalten der Weinsāure beim Erbitzen 40. Mikrochemische Unterscheidung von Welnsäure und Traubensăure 40. Relative I.öslichkeit von Ca-tartrat und Ca-razemat 40 . Brechweinstein 41.

Zitronensäure

Darstellung aus Zitrouen 41. Einwirkung von konzentrierter Schwefelsâre auf Zitronensäre 41. Bildung von Aceton aus Zitronensaure 41.

XVII. Chloralhydrat . . . . . . . . . . . . . . . . . .
Einwirkung von Wasser auf Chloral 42. Einwirkung von Kalilauge auf Chloralhydrat 42. Silbersplegelprobe auf Chloralhydrat 42. Blsulfit und Chloralhydrat 42.

XVIII. Aldehyd- und Ketonalkohole oder Kohlehydrate . . . .

Eigenschaften der Monosen. . . . . . . . . . . . . . FruLwse scher Lösung 43. Osazone 43.

Bildungsweisen der Monosen

Inversion von Saccharose (Rohrzucker) 43. Glyzerose 43.

Pentosen . . . . . . . . . . . . . . . . . . . . .

Furfurolreaktion 44.

Hexosen

Salzsăurereaktion 44. Calciumverbindung der d-Glukose 44. Glukoseschwefelsäure 45. Kupferverbindung der $d$-Glukose 45 . Nachweis der $d$-Glukose im Harn 45. Oxydation von $d$-Glukose mit Salpetersăure 45. Alkobolcharakter der $d$-Fruktose 45 . Nachweis von $d$-Fruktose 45. Oxydation der $d$-Galaktose zu Schleimsäure 46.

Biosen

Auffindung von Milchzucker in Milch 46. Laktosazon 46. „Bandzucker" 47. Unterscheidende Merkmale der Saccharose von den Monosen 47. Verhalten des Rohrzuckers beim Erhitzen 47. Tricalclumsaccharat 47. Inversion 47.

Polyosen . . . . . . . . . . . . . . . . . . . .

Stărkekleister 47. Unterscheidungsmerkmale der Stärke von den Monosen 47. Umwandlung von Stärke in $d$-Glukose 48 . Jodreaktion det Stărke 48. Baryumverbindung der Stärke 48 . Umwandlung von Stărke in Dextrin 48. Kartoffelstärke 49. Hydrolyse der Zellulose 49. Pergamentpapier 49. Löslichkeit von Zellulose in SoHwartzer schem Reagens 49. Nitrate der Zellulose 49. Nachweis von Ligoin in Papier 50.

\section{Eiweißkörper .}

Naohweis ron Stickstotf und schwefel in Eiweißstoffen 50. Aussalzen von EiweiB mit Ammoniumsulfat 60. Gerinnung von EiweiB durch Alkohol 51. Gerinnung von Eiweiß durch Salpetersaure 51. Biuretreaktion 51. MiLLON sches Reagens 51 . X anthoproteïnresktion 51 . Reaktion von ADAMXIEwicz 51. Zersetzungsprodukte des Keratins 51 .

XX. Cyanwasserstoff und seine einfachen und komplexen Salze . Cyanwasserstoff 52. Berliner Blau-Reaktion 52. Sllbercyanid 52. Hydrolyse von Cyankalium 52 . Uberfohrung von Ammoniumformiat in Cyauwasserstoff 62. 
Doppelferrocyanid von Kalium und Calcium 63. Ferrocyanwasserstoffsăure 53. Einwirkung von konzentrierter Schweielsäure auf Ferrocyankalium 53. Vorhalten vou Ferrocyankalium beim Erhitzen 53. Ferricyankalium 53. Reduktion von Ferricyankalium 54. Oxydation von Ferrocyankalium mit Kaliumpermanganat 54 .

\section{Kaliumcyanat und Kaliumthiocyanat}

Kaliumcyanat 5. Kaliumthiocyanat 54. Rhodsnsilber 5́.

XXII. Abkömmlinge der Kohlensäure . . . . . . . . . . 55

Schwefelkohlenstoff .

Brennbarkeit 55. Flüchtigkeit 55. Lösungsvermögen 6j. Baryumtrithiokarbonat 55. Kalium- und Kupfersalze der Xanthogensăure 56.

\section{Harnstoff}

Synthese von WÖLEk $b$ b. Oxalsaurer Harnstult 66. Salpetersaurer llarnsto 15 aus Harn 56. Methode von KNOP zur Bestimmung des Harnstoffs im Harn 57. Zersetzung des Harnstoffs durch Basen 67. Methode von Lienio zur Bestimmung des Harnstoffs 57. Verhalten des Harnstoffs beim Erbitzen 57. Cyanursăure 58.

Karbaminsäure

Calciumkarbamat 58. Ammoniumdithiokarbamat 68.

XXIII. Harnsäuregruppe .

Harnsaures Ammonium 59. Neutrales harnssures Kallum 59. Murexidreaktion der Harnsăure 59. Reduktion von Silbernitrat durol Harngtüu 59. Nurexidprobe auf Kaffeïn 59.

XXIV. Benzol und seine Homologen

Bestăndigkeit des Benzols gegenüber Halogenen 60. Abwesenheit doppeller Bindung in Benzol 60. Nitrobenzol 60. Benzolmonosulfosăure 60. Reaktion von Frisdel und Crabts 61. Bildung von Benzol aus benzoesaurem Kalk 61 . Oxydation von Seitenketten 61 .

XXV. Monohalogenverbindungen

Beständigkeit des Monochlorbenzols gegen alkoholisches Kali oder Natron 61 .

XXVI. Einwertige Phenole.

Darstellung von Phenol aus saiizglsaurem Calcium 62. Darstellung von Phenol durch Schmelzen von benzolsulfosaurem Natron mit Atzuatron 62. Natriumphenolat 63. Tribromphenol 63. Eisenchloridreaktion des Phenols 63. Erniedrigung des Schuelzpunktes von Phenol 63. Einwirkung von Salpetersłure auf Phenol 63. Kresol 63.

XXVII. Monoamidoverbindungen und ihre Derivate . . . . .

Anilin 64. Neutrale Resktion des Anilins 64. Anilinsalze 64. 'Tribromanilin 64. Chlorkalkreaktion des Anilins 64. Kaliumbichromatreaktion des Anilins 65. Acetanilld 65. Karbylaminreaktion 65. Diphenylamin 65. Nitrosodimethylanllin 65. LIEBERMANN s Probe auf aromstischen Nitrosoverblndungen 66. AzobenzoI, Hydrazobenzol und Benzidinsulfat 66. Diphenglharnstoff 66 .

XXVIII. Diazoverbindungen und Hydrazine . . . . . . . .

Benzoldiazoniumchlorid 67. Ersatz der $\mathrm{N}_{2}$-Gruppe im Benzoldiazoniumchlorid durch Hydroxyl 67. GatTkRsIANN s Reaktion 67. Diazoamidolienzol 67. Anidoa\%obenzol 67. Salzsaures Sal\% des L'henylhydrazins 68 . Oxydation des Phenylhydrazins mit FriLiso scher Lösung 68. 
XXIX. Benzoesäure und ibre Abkömmlinge . . . . . . . . 68

Benzonitril und Benzoesăure 68. Benzoesăure aus Benzoeharz 69. Fiūchtigkeit der Benzoesăure mit Wasserdampf 69. Löslichkeit in Wasser 69. Benzoesăureăthylester 69 . Benzanilid 69 . Benzamid 70 .

XXX. Benzaldehyd .

Silberspiegelprobe 70. Benzaldehydphenylbydrazon 70 . Bisulfitverbindung des Benzaldehyd 70 . Einwirkung von alkoholiscber Kaliauge auf Benzaldehyd 71 . Oxydation des Benzaldehyds durch die Luft 71 . Durch Kaliumpermanganat 71 .

XXXI. Benzolbomologe mit substituierten und mit ungesättigten Seitenketten .

Verseifuug von Benzylchlorid mit Kali- oder Natronlauge 72. Benzyljodid 72. Wirkung des Lichtes auf Toluol + Brom 72 . Verharzung von Benzylalkohol 72. Oxydation von 'Limtsäure mit Kaliumpermanganat 72 .

XXXII. Benzolderivate mit mehreren Substituenten . . . . .

Beweglichkeit des Halogens in Halogen-nitroverbindungen 73 . m-Dinitrobenzol 73 . Reaktionsfähigkeit eincr der Nitrogruppen von 1, 3, 5-Trinitrobenzol 73 . Nitrophenole 73. Gefärbte Salze von p-Nitrophenol 74. Pikrinsäure 74. Kaliumpikrat 74. Gesclunack der Pikringäure 74. Fürben mit Pikrinsăure it. Iso. purpursäure 74. Chlorpikrin 74. Ionisation der Pikrinsäure in wäBriger Lösung 74. Reaktion auf Brenzkatechin, Resorzin und Hydrochinon 75. Reduzierende Wirkuug der Dioxybenzole 75. Pyrogallol 75. Benzochinon 76. Chinhydron 76. Unterschied in Basizität der drei Nitraniline 76. Reaktionsfäbigkeit der Aminogruppe in Pikramid 76. p-Diazobenzolsulfosäure 76. Oxydation der Aminopbenole 77. Keaktion auf salpetrige Säure mit w-Phenylondiamin 77. Färben mit Amidoazobenzol 77. Reduktion des Amidoazobenzols 77. Ge8chmack des Saccharins 7 . Salizylsäure 77 . Einwirkuog von Bromwasser suf Salizylsăure 7 . Nachweis von Salizylsäure und Phenol 78 . Calciumsalizylat $i \varepsilon$. Tinte 78 . Einwirkung von Tannin auf Gelatine 78 . Reaktion auf Tannin 78. Einwirkung von Tannin auf Chinin 78. Reaktion zwischen Albumin und Tannin 79. Zersetzung der Antbranilsăure 79. Phtalsăureanhydrfd 79. Fluoreszeïn 79. Phenolphtaleïn 79. Chinoide Form des Phenolphtsleïns 79 .

XXXIII. Terpene und Kampfer.

Künstlicher Kampfer 80. Flüchtigkeit des Kampfers mit Wasserdampf 80 . Kampfer und Wasser 80. Kampferaäure 80.

XXXIV. Benzolkerne, die durch Kohlenstoff miteinander verbunden sind

Rosolsäure 81. Fucbsin 81. Einwirkung von konzentrierter Salzsäure auf eine Fucbsinlősung 81 . Farbbase 81 . 1.eukobase 81 .

XXXV. Kondensierte Benzolkerne . . . . . . . . . . .

Verhalten des Naphtalins beim Erhitzen 82. Naphtalinpikrinsäure 82. Napbtalinmonosulfosăuren 82. $a$-Nitronaphtalin 82. Naphtol 82. Martiusgelb 82. Naphtylamine 8?. Kongorot 83. Naphtionsäure 83. Oxydation von Anthracen nit Ciromsäure 83. Reaktion auf Anthrachinon 83. Kaliumsalz von Alizarin 83.

XXXVI. Heterozyklische Verbinduugen .

Pyridin 84. Einwirkung von Pyridin auf Eisenchlorid 84. Einwirkung von I'yridin auf Quecksilberchlorld \&5̃. Nachweis von Pyridin 84. verhalten des Pyridins $z u$ den Alkaloidreagention S4. Beständigkeit des Pyridins gegen BAEYER sches Reagenz 81. Beständigkeit des Pyridins gegen Oxydationsmittel 85. Pyrrolrot 85̃. Indopheninreaktion 85. Nachweis von Antipyrin 85. 
XXXVII. Kondensationsprodukte von Benzol mit heterozyklischen Kernen . . . . . . . . . . . . . . . . . . . . 85

Skraup sche Synthese des Chinolins 85. Salzsaures Chinolin 86. Chlnoliupikrat 86. Chinolinbichromat 86. Flüchtigkeit des Indigos 86. Löslichkeit von Indigo in Nitrobenzol 86. Indigosulfosaure 86. Oxydation von Indigo und Isatin 86. „Indigoküpe" 86.

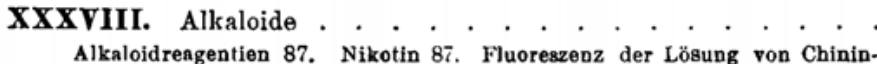
sulfat 87. Nachweis von Cbinin 87. Nachweis voc Strycbnin 87. Nachweis von Brucin s8.

Register . . . . . . . . . . . . . . . . . . . . . 8 